\title{
Dielectric constant of a three-dimensional woven glass fibre composite: analysis and measurement
}

DOI:

10.1016/j.compstruct.2017.08.061

\section{Document Version}

Accepted author manuscript

Link to publication record in Manchester Research Explorer

\section{Citation for published version (APA):}

Li, Z., Haigh, A., Soutis, C., Gibson, A., \& Sloan, R. (2017). Dielectric constant of a three-dimensional woven glass fibre composite: analysis and measurement. Composite Structures.

https://doi.org/10.1016/j.compstruct.2017.08.061

\section{Published in:}

Composite Structures

\section{Citing this paper}

Please note that where the full-text provided on Manchester Research Explorer is the Author Accepted Manuscript or Proof version this may differ from the final Published version. If citing, it is advised that you check and use the publisher's definitive version.

\section{General rights}

Copyright and moral rights for the publications made accessible in the Research Explorer are retained by the authors and/or other copyright owners and it is a condition of accessing publications that users recognise and abide by the legal requirements associated with these rights.

\section{Takedown policy}

If you believe that this document breaches copyright please refer to the University of Manchester's Takedown Procedures [http://man.ac.uk/04Y6Bo] or contact uml.scholarlycommunications@manchester.ac.uk providing relevant details, so we can investigate your claim.

\section{OPEN ACCESS}


Dielectric constant of a three-dimensional woven glass fibre composite: analysis and measurement Zhen $\mathrm{Li}^{1}$, Arthur Haigh ${ }^{2}$, Constantinos Soutis ${ }^{1 *}$, Andrew Gibson ${ }^{3}$ and Robin Sloan ${ }^{2}$

${ }^{1}$ Aerospace Research Institute, The University of Manchester, Manchester, M13 9PL, UK

${ }^{2}$ School of Electrical and Electronic Engineering, The University of Manchester, Manchester, M13 9PL, UK

${ }^{3}$ Faculty of Science and Engineering, Manchester Metropolitan University, Manchester, M1 5GD, UK

*Corresponding author: constantinos.soutis@manchester.ac.uk

\begin{abstract}
This paper presents a novel methodology for predicting the dielectric constant of three-dimensional woven glass fibre-reinforced composites. A well-established approach of deriving the effective dielectric constant is the dielectric mixing formulae (rule of mixtures based), which either provide a single value or offer upper and lower bounds. For composites with three-dimensional fibre architecture, an accurate model considering the three-dimensional effect is needed. Here, the anisotropic effect is revealed using electromagnetic simulation to extract the effective dielectric constant of a model material with unidirectional fibres, which are aligned or orthogonal to the electric field. The rule of mixtures based formulae are evaluated. The most suitable formula selected for each case is then extended to a general case with arbitrary fibre orientation and is further used to characterise the capacitor element of an electromagnetic model for 3D woven composites. The proposed method is compared to measurements to demonstrate the improved accuracy.
\end{abstract}

Keywords: Dielectric constant; glass fibre; 3D woven composites; simulation; modelling. 


\section{Introduction}

Glass fibre-reinforced polymer (GFRP) composites are widely used in aerospace, shipping, automotive industries and wind turbine blades, due to their numerous advantages such as high stiffness and strength characteristics, a high strength-to-weight ratio and better corrosion/chemical resistance [1-3]. Recently, the woven fabric composites have received increasing interest, as manufacturing costs are reduced and the impact damage resistance is significantly improved [4]. For example, in 3D woven composites, the warp yarns, weft yarns and Z-binders are highly interlaced in three directions resulting in the through-thickness reinforcement that prevents delamination hence improves damage resistance. It is also important to understand the electromagnetic (EM) characteristics for applications (e.g., airborne radomes and wind turbine blades [5]) where radar interference [6], lightning discharge and electromagnetic testing are all of interest. For dielectrics such as glass fibre composites the primary constituent parameter is the real part of the relative permittivity (also called dielectric constant, $\varepsilon_{\mathrm{r}}^{\prime}$ ), as the dielectric loss (evaluated by loss tangent, $\tan \delta$ ) is minimal and the magnetic permeability is equal to that of free space.

Previous studies have considered how $\varepsilon_{\mathrm{r}}^{\prime}$ varies as a function of fibre volume fraction. A number of rule of mixtures based formulae have been developed, such as Wiener limits, Maxwell Garnett formula and Looyenga formula [7]. In these closed-form formulae, the effective dielectric constant is a function of the dielectric constants of the fibre and resin. The upper and lower bounds of the effective dielectric constant can be provided by Wiener upper limit and Wiener lower limit, respectively [8]. The limits were given based on an analysis of laminated structures, which corresponded to capacitors connected in series or in parallel with respect to the applied electric field. However, most practical cases are between the two limits, and the search for accurate models for these intermediate cases has drawn considerable attention in the dielectric constant research community. 
An alternative equivalent lumped circuit model introduced by Chin et al. $[9,10]$ was built for unidirectional and multidirectional laminates, where a lamina was modelled as parallel lumped resistor-capacitor circuit. This method accurately predicted the data obtained from free space measurement over X-band (8-12 GHz). Yao et al. [11] extended this circuit model to 3D orthogonal woven composites. In the modelling, the effects of the warp yarns and Z-yarns that were both orthogonal to the electric field were assumed the same. The simulation results did not agree well with experiments.

With 3D weaves, the permittivity becomes more anisotropic, so the effect of fibre direction should be thoroughly studied. In this paper, we introduce an EM pre-processing approach that investigates the permittivity when the fibre directions are orthogonal. A composite model with unidirectional fibres is built in a waveguide section. The scattering parameters obtained from simulation are used to extract the effective dielectric constant, and this is repeated for a range of fibre volume fractions. These basic models are then compared to the results produced by a set of mixing formulae, and for each fibre direction a particular formula is selected. This recommendation is then used to develop a capacitor model of a 3D woven composite structure from which the overall dielectric constant is estimated. This cycle is completed for a number of practical setups and the predicted results compare well with the experiment.

\section{Evaluation of the effect of fibre directions}

\subsection{Numerical simulation}

The dielectric constant of the fibre-resin mixture is dependent on the angle between the fibre orientation and the electric field of the incident electromagnetic wave [12]. This polarisation effect is investigated by numerical simulation using $\mathrm{CST}^{\circledR}$ software. As shown in Figure 1(a), a square unit cell made up of a circular cross-sectional fibre and resin is employed here to represent the microstructure of the yarn. The dielectric properties of both fibre and resin are listed in Table 1. 
Table 1 Dielectric properties of the fibre and resin used for investigation of the polarisation effect

\begin{tabular}{ccc}
\hline Dielectric properties & E-glass fibre [13] & 510 Resin [13] \\
\hline Dielectric constant & 6.20 & 3.00 \\
Loss tangent & $1.50 \times 10^{-3}$ & $1.67 \times 10^{-2}$
\end{tabular}

Assuming the size of the unit cell is $\mathrm{L}$ and the diameter of the fibre is $\mathrm{D}$, the fibre volume fraction $v_{f}$ is written as

$$
v_{f}=\frac{\pi D^{2}}{4 L^{2}}
$$

As illustrated in Figure 1 (b), the electric field of the incident EM waves with $\mathrm{TE}_{10}$ mode is in the $\mathrm{Y}$ direction and the waves propagate along the $\mathrm{Z}$ direction. Three representative cases are considered, i.e., the fibre direction parallel to the electric field (Case 1, Figure 1 (c)), orthogonal to the electric field (Case 2, Figure 1 (d)) and parallel to the propagation direction (Case 3, Figure 1 (e)). The cuboids for the three cases are modelled within the X-band waveguide, the inner dimensions of which are $22.86 \mathrm{~mm}\left(\mathrm{a}^{\prime}\right) \times 10.16 \mathrm{~mm}\left(\mathrm{~b}^{\prime}\right)$. X-band is chosen as this range is widely used for navigation radars [14].

$\mathrm{L}$ is set to be $2.54 \mathrm{~mm}\left(1 / 9\right.$ of $\mathrm{a}^{\prime}$ and $1 / 4$ of $\left.\mathrm{b}^{\prime}\right)$ for easy implementation. In each case, $v_{f}$ is $60 \%$. As seen in Figure 1(f), the thickness along the waveguide (t) is $2.54 \mathrm{~mm}$, and Port 1 and Port 2 are electromagnetically distant from the sample with air gaps of $50 \mathrm{~mm}$.

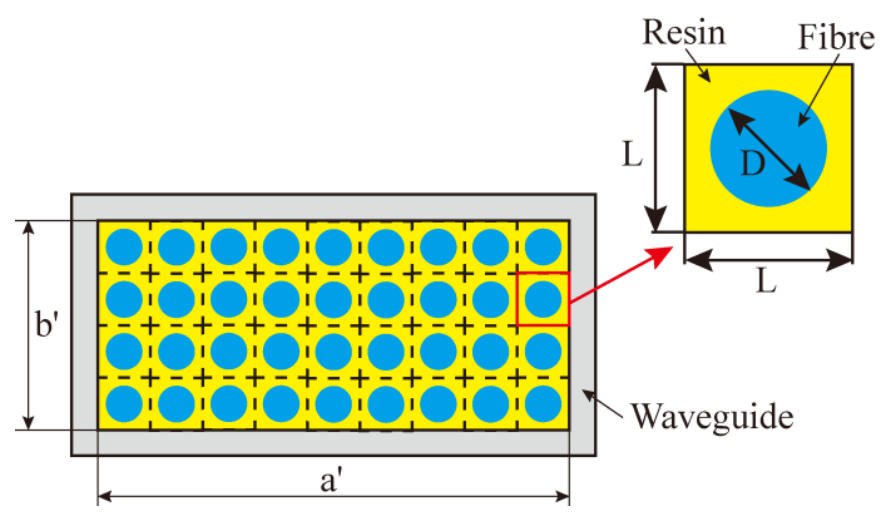

(a) unit cell of the fibre-resin mixture 


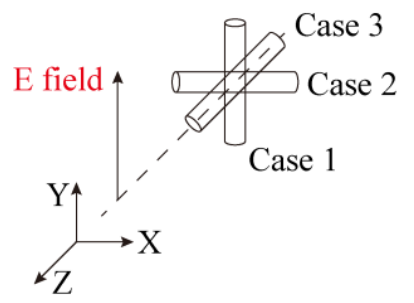

(b) Diagram of the three cases investigated

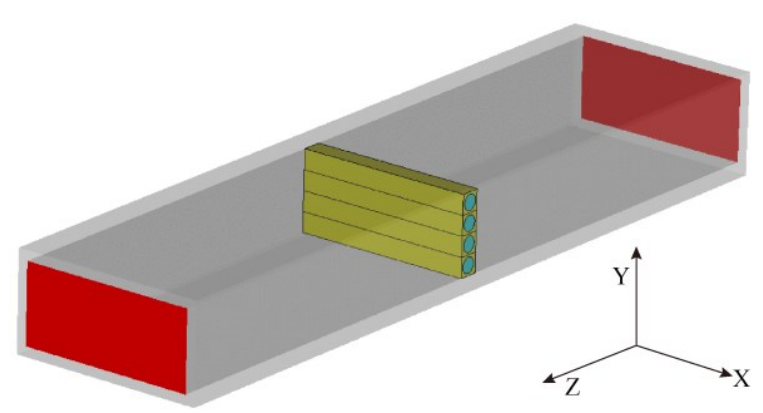

(d) Case 2

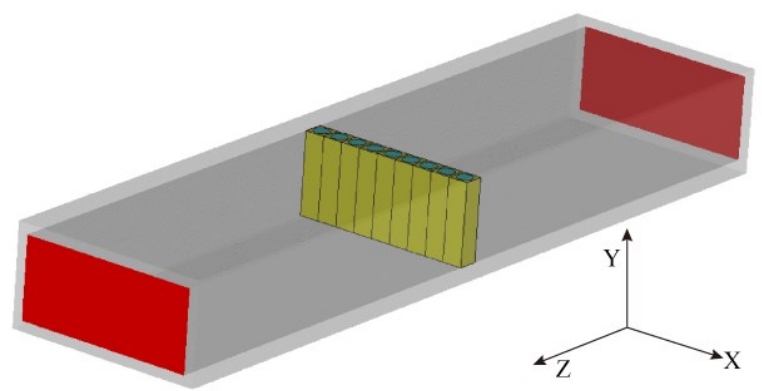

(c) Case 1

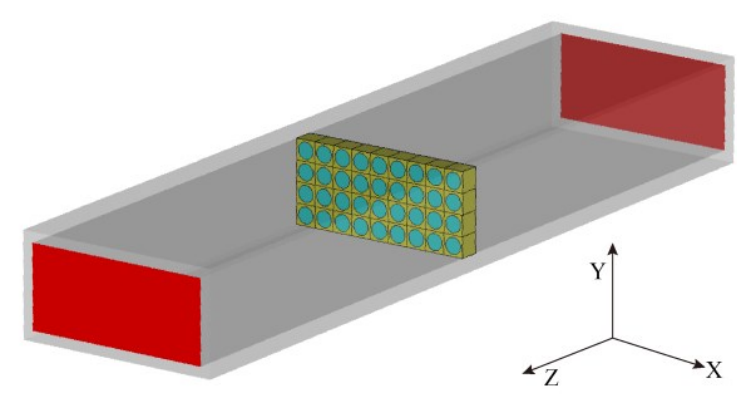

(e) Case 3

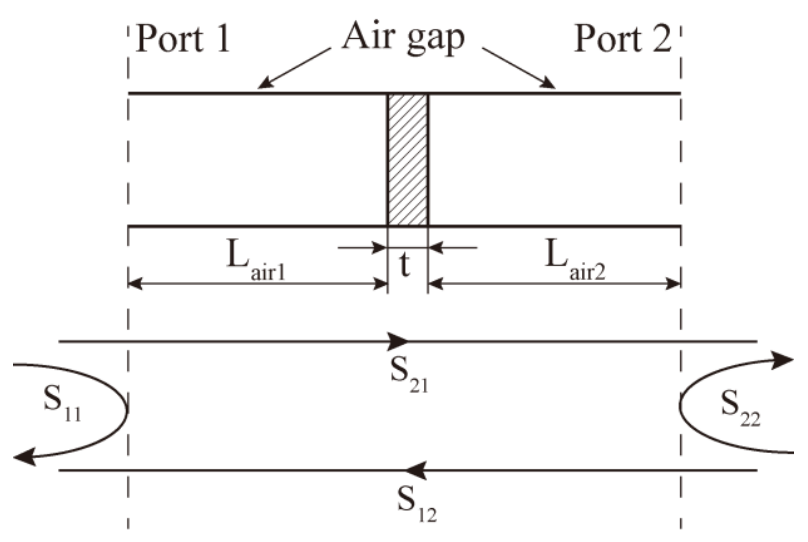

(f) Cross section of the waveguide cell

Figure 1 Three representative cases for the study of the effects of fibre direction

The simulation results presented in Figure 2 demonstrate that the scattering parameters (reflection coefficients $S_{11}$ and transmission coefficients $S_{21}$ ) are significantly affected by fibre directions. And some of the assumptions made in the literature are incorrect, i.e., Case 2 and Case 3 are electromagnetically equivalent [11]. 


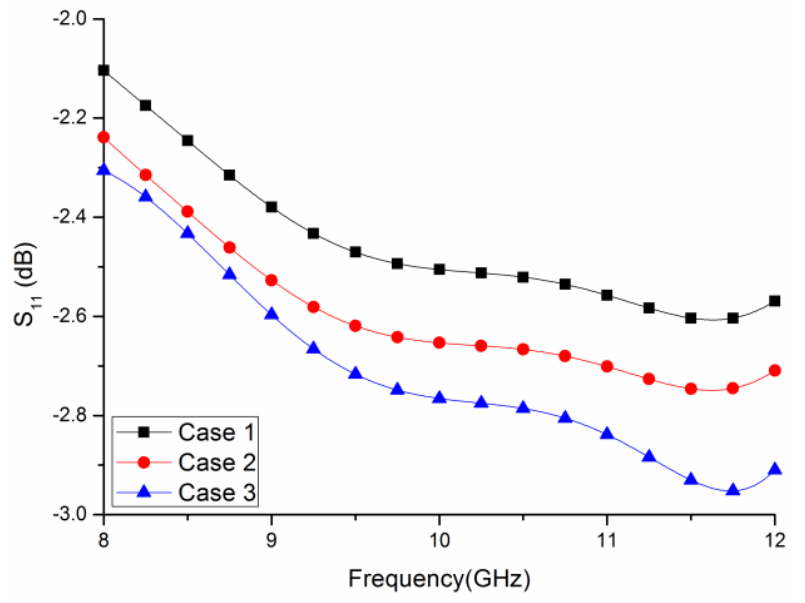

(a) $\mathrm{S}_{11}$-Magnitude

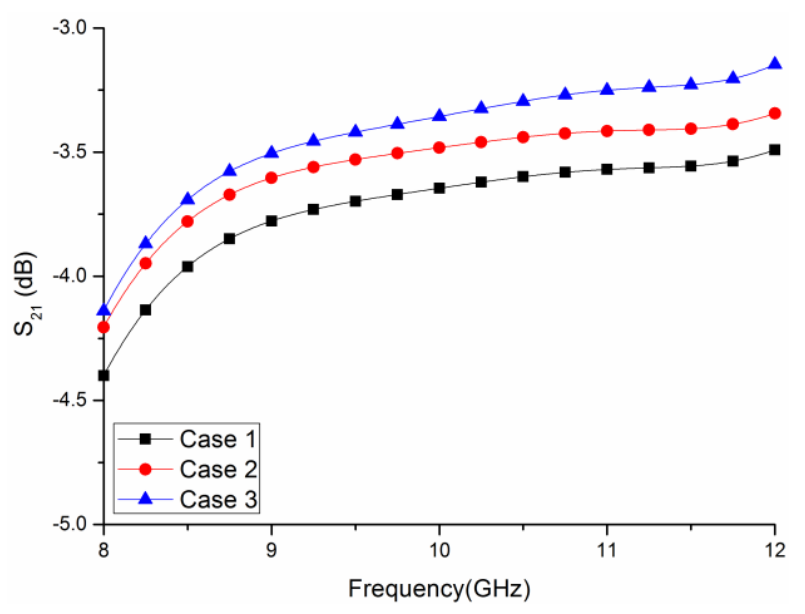

(c) $\mathrm{S}_{21}$-Magnitude

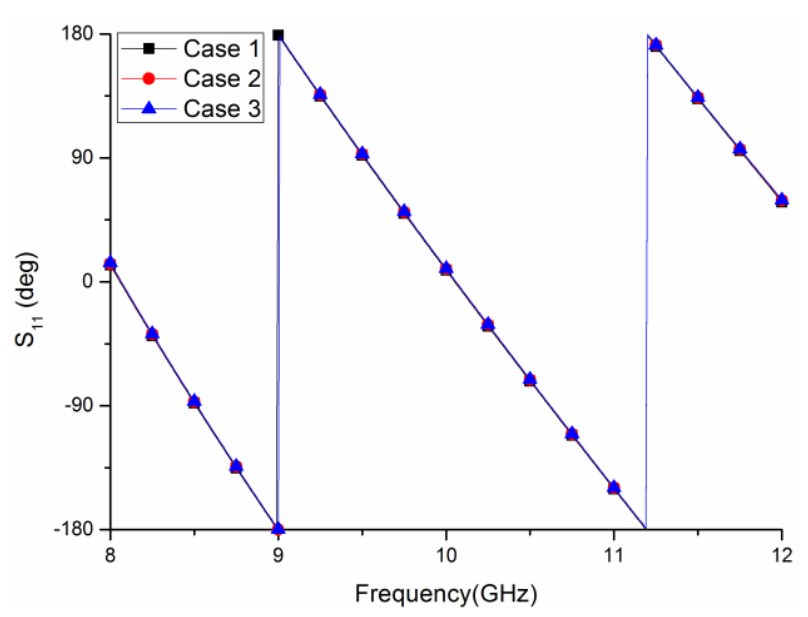

(b) $\mathrm{S}_{11}$-Phase

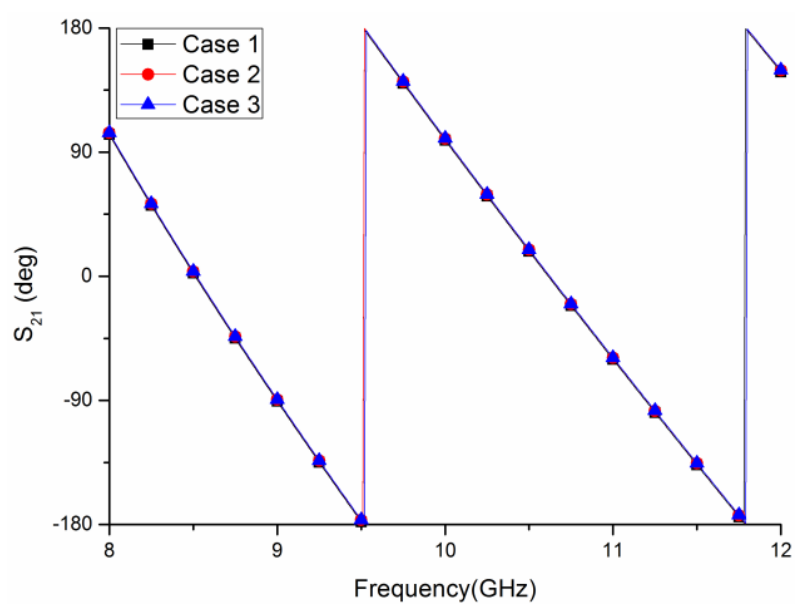

(d) $\mathrm{S}_{21}$-Phase

Figure 2 Variations of $\mathrm{S}_{11}$ and $\mathrm{S}_{21}$ versus frequencies in the three cases with orthogonal fibre directions

\subsection{Calculation of the effective permittivity from the scattering parameters}

The Tischer model [15] can be adopted for evaluation. Assuming there is only one sample layer with a thickness of $\mathrm{t}$, the expressions for $\mathrm{S}_{11}$ and $\mathrm{S}_{21}$ are:

$$
\begin{aligned}
& {\left[\mathrm{S}_{11}\right]_{\text {fibre-resin }}=\frac{-2\left(\gamma_{0}^{2}+\beta_{0}^{2}\right) \sinh \gamma_{0} t}{2\left(\gamma_{0}^{2}-\beta_{0}^{2}\right) \sinh \gamma_{0} t+4 j \gamma_{0} \beta_{0} \cosh \gamma_{0} t}} \\
& {\left[\mathrm{~S}_{21}\right]_{\text {fibre-resin }}=\frac{4 j \gamma_{0} \beta_{0}}{2\left(\gamma_{0}^{2}-\beta_{0}^{2}\right) \sinh \gamma_{0} t+4 j \gamma_{0} \beta_{0} \cosh \gamma_{0} t}}
\end{aligned}
$$


where $\beta_{0}=2 \pi / \lambda_{\mathrm{g}}$ is the phase constant of the transmission line. $\gamma_{0}$ is the complex propagation constant. $\lambda_{\mathrm{g}}$ is the guide wavelength of the empty waveguide.

$$
\begin{gathered}
\gamma_{0}=j 2 \pi \sqrt{\frac{\varepsilon_{\mathrm{r}}}{\lambda^{2}}-\frac{1}{\lambda_{c}^{2}}} \\
\lambda_{g}=\frac{1}{\sqrt{\frac{1}{\lambda^{2}}-\frac{1}{\lambda_{c}^{2}}}}=\frac{\lambda}{\sqrt{1-\left(\frac{\lambda}{\lambda_{c}}\right)^{2}}}
\end{gathered}
$$

where $\lambda$ and $\lambda_{c}=2 \mathrm{a}^{\prime}$ are the free space wavelength and cut-off wavelength, respectively. The NewtonRaphson approximation method is used to obtain the unknown complex propagation constant $\gamma_{0}$ from the complex transcendental equations:

$$
\begin{aligned}
& {\left[\mathrm{S}_{11}\right]_{\text {CST }}-\left[\mathrm{S}_{11}\right]_{\text {fibre-resin }} e^{-2 j \beta_{0} \mathrm{~L}_{\text {airl }}}=0} \\
& {\left[\mathrm{~S}_{21}\right]_{\text {CST }}-\left[\mathrm{S}_{21}\right]_{\text {fibre-resin }} e^{-j \beta_{0}\left(\mathrm{~L}_{\text {air } 1}+\mathrm{L}_{\text {air }}\right)}=0}
\end{aligned}
$$

where $L_{a i r 1}$ and $L_{a i r 2}$ are the lengths of the air gaps shown in Figure 1 (f). Here, the S-parameters obtained from CST simulation are phase shifted due to the presence of the air gaps.

Finally, the effective permittivity $\left(\varepsilon^{*}\right.$ eff $)$ can be obtained from

$$
\varepsilon_{\text {eff }}^{*}=\lambda^{2}\left[\left(\frac{\gamma_{0}}{2 \pi}\right)^{2}+\frac{1}{\lambda_{c}^{2}}\right]=\varepsilon_{r}^{\prime}(1-j \tan \delta)
$$

As mentioned above, only the real part of the relative effective permittivity (i.e., dielectric constant) is of interest in the following analysis. Two limiting cases with either $100 \%$ glass fibre or resin are calculated to verify the accuracy of the permittivity extraction approach. As presented in Figure 3 (a), it is indicated that there is good agreement between the simulation and the real values (Table 1). In each representative case, the dielectric constant slightly increases with increasing frequency. The highest values exist in Case 1, which is followed by Case 2 and Case 3. It is noted again that the results of Case 2 and Case 3 differ significantly. The variation of the dielectric constant with respect 
to $v_{f}$ is shown in Figure $3(\mathrm{~b})$. At each $v_{f}$, the dielectric constant values are averaged over the frequency range. The curves for Case 2 and Case 3 overlap when $v_{f}$ is less than $20 \%$. When $v_{f}$ is above $60 \%$, the curve for Case 2 becomes close to that for Case 1.

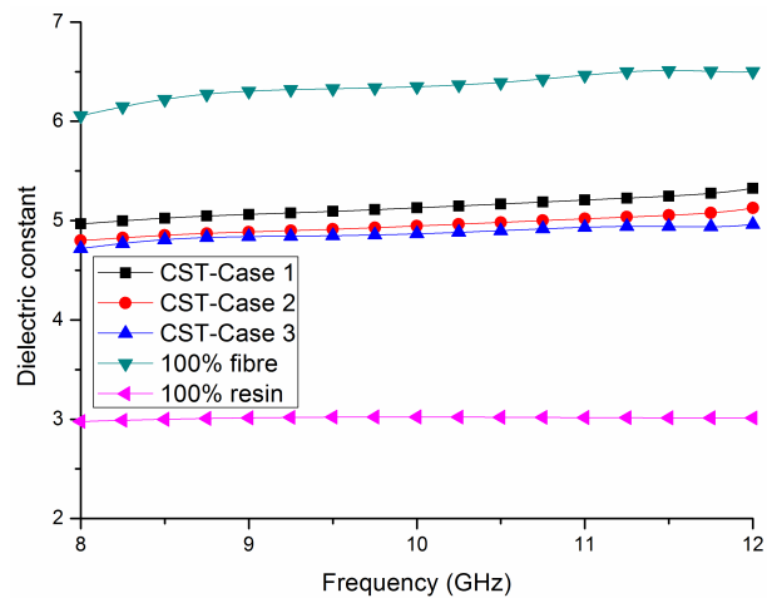

(a) The simulation results for two limiting cases and the three representative cases with $v_{f}=60 \%$

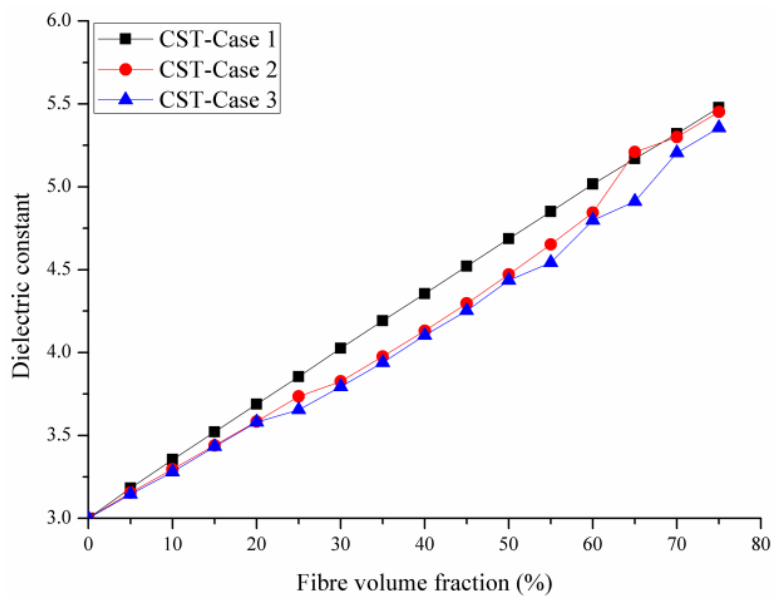

(b) Variation of the dielectric constant with respect to $v_{f}$

Figure 3 Comparison of the dielectric constants of the three representative cases

2.3 Comparison between simulation results and the rule of mixtures based formulae

Mixing formulae are based on relative volumes of fibres and resin. A number of formulae have been reported in the literature and are reproduced below for completeness:

Wiener upper limit:

Wiener lower limit:

Hashin Shtrikman upper bound: $\quad \varepsilon^{\prime}=\varepsilon_{f}^{\prime}+\frac{1-v_{f}}{\frac{v_{f}}{3 \varepsilon_{f}^{\prime}}-\frac{1}{\varepsilon_{f}^{\prime}-\varepsilon_{m}^{\prime}}}$

Hashin Shtrikman lower bound:

$$
\varepsilon^{\prime}=v_{f} \varepsilon_{f}^{\prime}+\left(1-v_{f}\right) \varepsilon_{m}^{\prime}
$$


Maxwell Garnett mixing rule: $\quad \varepsilon^{\prime}=\varepsilon_{m}^{\prime}+\frac{3 v_{f} \varepsilon_{m}^{\prime}}{\left[\left(\varepsilon_{f}^{\prime}+2 \varepsilon_{m}^{\prime}\right) /\left(\varepsilon_{f}^{\prime}-\varepsilon_{m}^{\prime}\right)\right]-v_{f}}$

Looyenga formula:

$$
\varepsilon^{\prime \frac{1}{3}}=v_{f} \varepsilon_{f}^{\prime \frac{1}{3}}+\left(1-v_{f}\right) \varepsilon_{m}^{\prime \frac{1}{3}}
$$

where $\varepsilon_{f}^{\prime}$ and $\varepsilon_{m}^{\prime}$ are the dielectric constants of the fibre and resin, respectively.

The equation for Hashin Shtrikman lower bound is identical to the Maxwell Garnett mixing rule. In the circuit model proposed by Chin [9], the expression for Y-directed fibre (Case 1) is the same as Wiener upper limit. And the formula for X-directed fibre (Case 2) is

$$
\varepsilon^{\prime}=\frac{4 \varepsilon_{m}^{\prime 2}+\pi \varepsilon_{m}^{\prime} \sqrt{v_{f}}\left(\varepsilon_{f}^{\prime}-\varepsilon_{m}^{\prime}\right)}{4 \varepsilon_{m}^{\prime}+\pi\left(\varepsilon_{f}^{\prime}-\varepsilon_{m}^{\prime}\right)\left(\sqrt{v_{f}}-v_{f}\right)}
$$

As shown in Figure 4, the dielectric constant is underestimated by the Wiener lower limit and the circuit model for Case 2, while the other expressions are close to the CST simulation results.

Specifically, the results provided by the Wiener upper limit, Hashin upper bound and Looyenga formula agree with those by simulation for Case 1, Case 2 and Case 3, respectively. Therefore, these three expressions can be used to effectively evaluate the dielectric constants of the three elementary cases with unidirectional fibres.

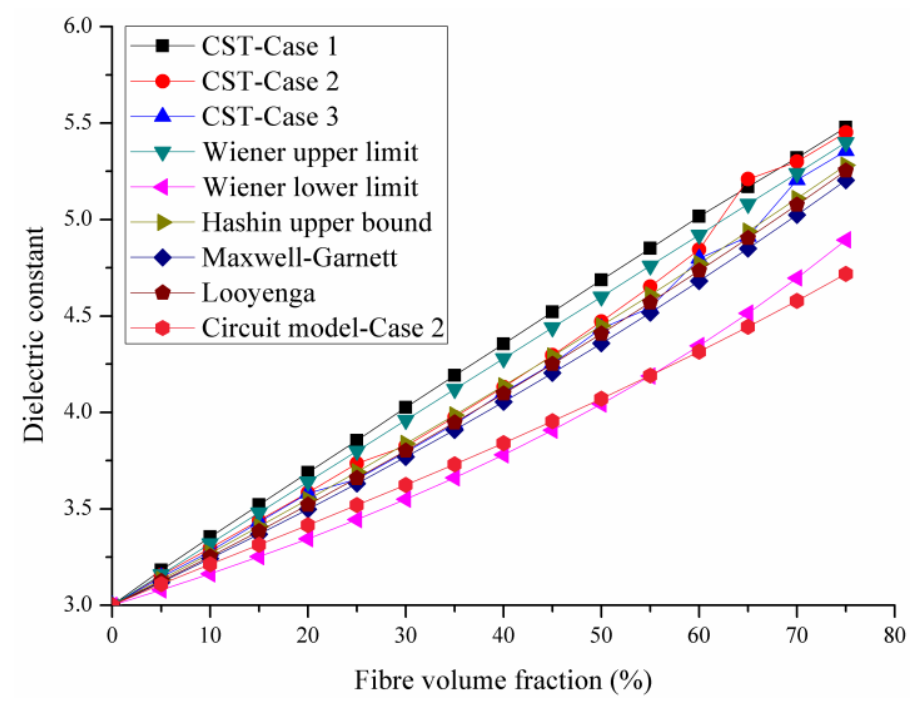

Figure 4 Comparison of the predicted dielectric constants given

by the simulation and the rule of mixtures based formulae 
2.4 Verification of the recommendation for dielectric constant calculation

The recommendation of using the Wiener upper limit for the model with Y-directed fibres, Hashin upper bound for the model with X-directed fibres and Looyenga formula for the model with Zdirected fibres was based on one type of fibre-resin specified in [13]. It is worth investigating the effect of changing the fibre-resin type on this recommendation/result. The dielectric properties of an alternative fibre-resin set are listed in Table 2 . The fibre volume fraction is $60 \%$ as well.

Table 2 Dielectric properties of another set of glass fibre and resin used for verification

\begin{tabular}{ccc}
\hline Dielectric properties & ECR glass fibre [16] & Epoxy resin [11] \\
\hline Dielectric constant & 7.0 & 3.0 \\
Loss tangent & $3.1 \times 10^{-3}$ & $2.0 \times 10^{-2}$
\end{tabular}

As demonstrated in Table 3, reasonable accuracy can be obtained by the proposed strategy. The error of $-5.10 \%$ for Case 1 is more acceptable than the error of $30 \%$ that would result if the Wiener lower limit were used.

Table 3 Comparison of the effective dielectric constants calculated from simulation and the three selected formulae for another set of glass fibre and resin

\begin{tabular}{ccccccc}
\hline \multirow{2}{*}{$\begin{array}{c}\text { Method } \\
\text { Value 1 }\end{array}$} & Error (\%) & Value & Error (\%) & Value & Error (\%) \\
& Vase 2 & & \multicolumn{2}{c}{ Case 3 } \\
\hline CST simulation & 5.48 & - & 5.20 & - & 5.11 & - \\
Wiener upper limit & 5.40 & -1.56 & & & & \\
Hashin upper bound & & & 5.19 & -0.23 & & \\
Looyenga formula & & & & 5.13 & 0.38 \\
\hline
\end{tabular}

\subsection{General case with non-orthogonal fibre orientations}

In practice, for actual weaves fibre directions will generally not be orthogonal. A transformation has been used in the literature for 2D generalisation [17] and here this is extended for 3D modelling. As 
shown in Figure 5, if the angle between the fibre direction and $\mathrm{X}$ axis is $\theta$ and the angle between the fibre direction and X-Y plane is $\varphi$, the effective dielectric constant tensor $\left[\varepsilon^{\prime}\right]^{(\theta, \varphi)}$ can be given by

$$
\left[\varepsilon^{\prime}\right]^{(\theta, \varphi)}=\left[\begin{array}{ccc}
\cos \theta & \sin \theta & 0 \\
-\sin \theta & \cos \theta & 0 \\
0 & 0 & 1
\end{array}\right]\left[\begin{array}{ccc}
\cos \varphi & 0 & \sin \varphi \\
0 & 1 & 0 \\
-\sin \varphi & 0 & \cos \varphi
\end{array}\right]\left[\begin{array}{ccc}
\varepsilon_{\text {Case 2 }}^{\prime} & 0 & 0 \\
0 & \varepsilon_{\text {Case 1 }}^{\prime} & 0 \\
0 & 0 & \varepsilon_{\text {Case } 3}^{\prime}
\end{array}\right]\left[\begin{array}{ccc}
\cos \varphi & 0 & -\sin \varphi \\
0 & 1 & 0 \\
\sin \varphi & 0 & \cos \varphi
\end{array}\right]\left[\begin{array}{ccc}
\cos \theta & -\sin \theta & 0 \\
\sin \theta & \cos \theta & 0 \\
0 & 0 & 1
\end{array}\right]
$$

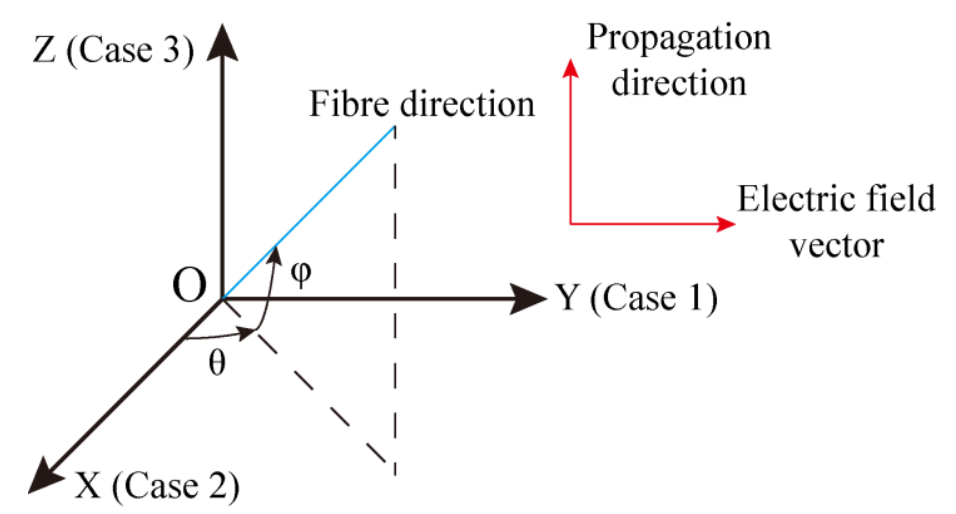

Figure 5 Schematic diagram of an arbitrary fibre direction with respect to the electric field vector and propagation direction in a $3 \mathrm{D}$ coordinate system

\section{Electromagnetic modelling of 3D woven glass fibre composites}

Here a 3D woven structure is meshed into finite elements. Different from the element types (e.g., bar, beam or shell) for mechanical analysis [18], the concept of a parallel-plate capacitor is employed to represent the dielectric characteristics of the material, which is illustrated in Figure 6. A real capacitor can be represented as a capacitance and a resistance in parallel [19]. The capacitance $C_{p}$ is written as:

$$
\mathrm{C}_{p}=\frac{\varepsilon_{0} \varepsilon_{r p}^{\prime} \mathrm{A}}{\mathrm{d}}
$$

where $\mathrm{A}$ is the area of the plate and $\mathrm{d}$ is the distance between the two parallel plates. $\varepsilon_{0}=8.854 \times 10^{-12}$ $\mathrm{F} \cdot \mathrm{m}^{-1}$ is the permittivity of free space, and $\varepsilon_{r p}^{\prime}$ is the effective dielectric constant of the in-between medium (e.g., the resin, Case 1, Case 2, Case 3 or general cases discussed above).

The resistance $\mathrm{R}_{p}$ is given by 


$$
\mathrm{R}_{p}=\frac{\mathrm{d}}{\sigma \mathrm{A}}
$$

where $\sigma$ is the conductivity of the dielectric material. However, the resistor can be viewed as open circuit, as the conductivities of both glass fibre $\left(\sim 10^{-10} \mathrm{~S} / \mathrm{m}[20]\right)$ and resin $\left(\sim 10^{-7} \mathrm{~S} / \mathrm{m} \mathrm{[21]}\right)$ are significantly low (hence the loss tangent of the material is negligible). Therefore, only the capacitance is considered here.

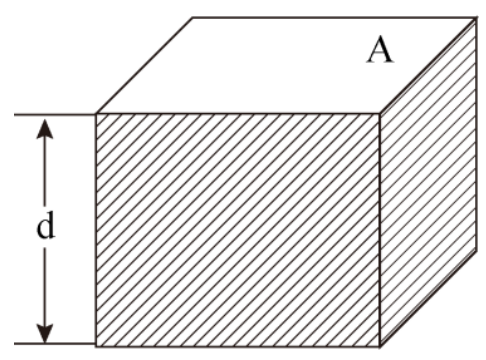

(a) A capacitor model used as the finite element

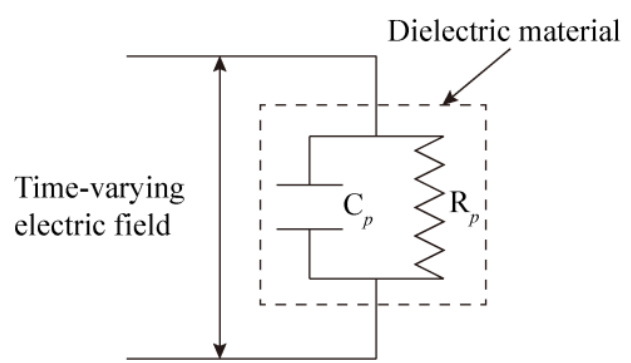

(b) Equivalent circuit model of the element

Figure 6 The parallel-plate capacitor model used as the finite element in the proposed electromagnetic modelling of $3 \mathrm{D}$ woven glass fibre composites

Based on the capacitor model, a 3D woven orthogonal structure is built as an example. As shown in Figure 7 (a), a representative volume element (RVE) [22] is represented by a matrix of $\mathrm{m} \times \mathrm{n} \times \ell$ capacitors (Figure 7 (b)). The plane perpendicular to the electric field is made up of $n \times \ell$ small areas, namely $A_{j k}, j=1,2, \ldots, n$ and $k=1,2, \ldots, \ell$. The thickness of the ith layer is denoted by $d_{i}, i=1$, $2, \ldots, \mathrm{m}$.

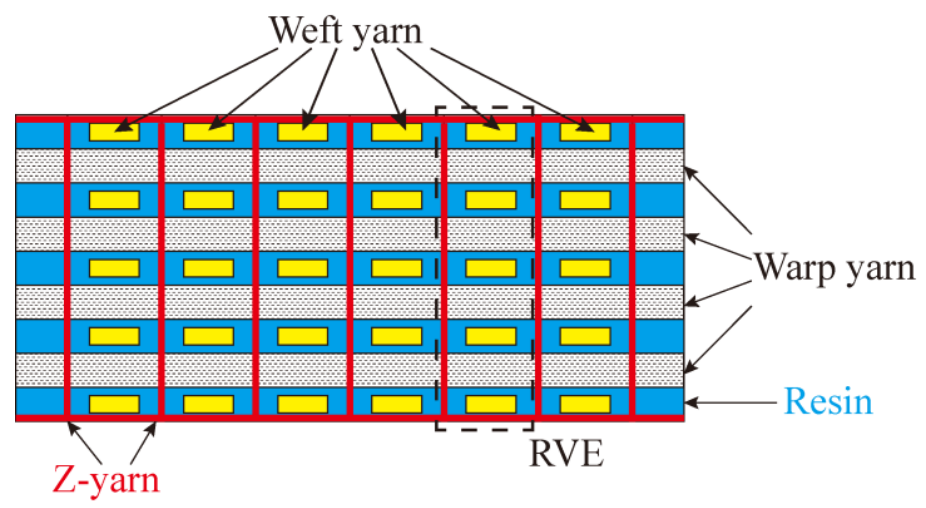

(a) RVE of a 3D orthogonal woven structure 


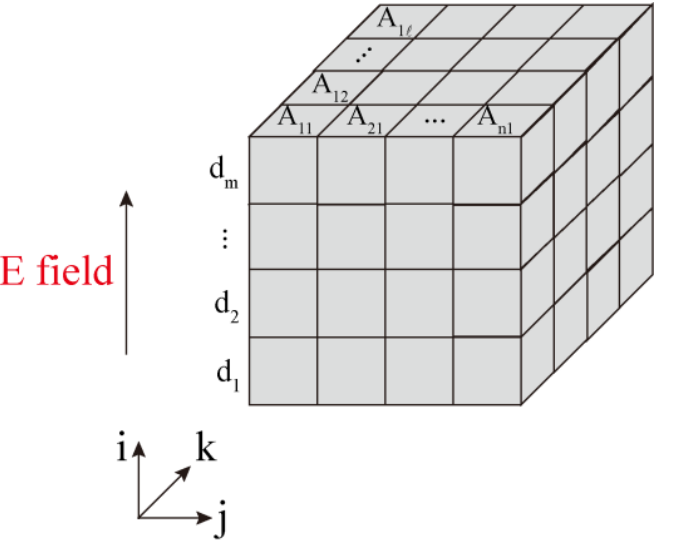

(b) Dimensions of each element in the RVE

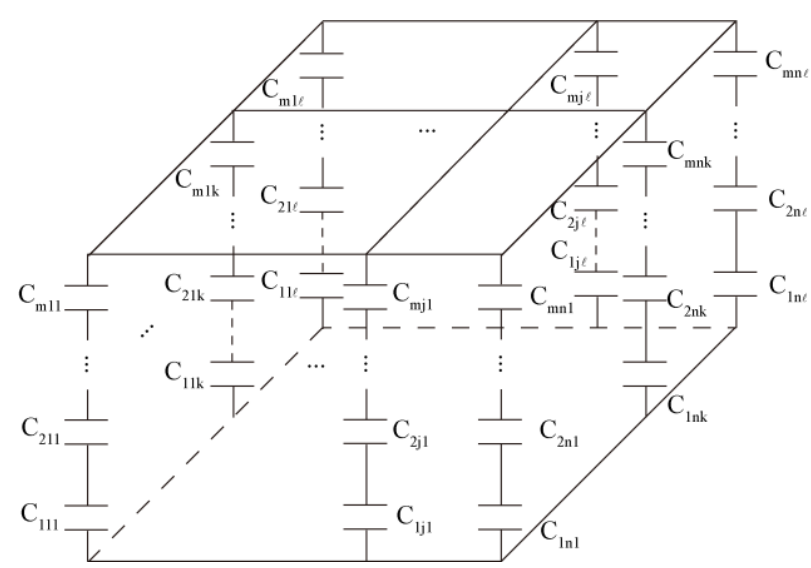

(c) Equivalent circuit model of the RVE

Figure 7 Proposed electromagnetic modelling of a 3D orthogonal woven composites According to Equation (14), the capacitance of each element $\mathrm{C}_{\mathrm{ijk}}$ can be rewritten as

$$
\mathrm{C}_{\mathrm{ijk}}=\varepsilon_{0} \varepsilon_{i j k}^{\prime} \frac{\mathrm{A}_{j k}}{d_{i}}
$$

Therefore, the total capacitance can be derived:

$$
\mathrm{C}=\sum_{j=1}^{n} \sum_{k=1}^{\ell}\left(\sum_{i=1}^{\prime} \frac{1}{\mathrm{C}_{\mathrm{ijk}}}\right)^{-1}
$$

The overall capacitance can also be represented by

$$
\mathrm{C}_{\mathrm{RVE}}=\varepsilon_{0} \varepsilon_{\text {eff }}^{\prime} \frac{\mathrm{A}_{\mathrm{RVE}}}{\mathrm{d}_{\mathrm{RVE}}}=\varepsilon_{0} \varepsilon_{\text {eff }}^{\prime} \frac{\sum_{j=1}^{n} \sum_{k=1}^{\ell} \mathrm{A}_{j k}}{\sum_{i=1}^{m} d_{i}}
$$

where $A_{R V E}$ is total area orthogonal to the electric field, and $d_{R V E}$ is the whole length along the electric field. The effective dielectric constant of the 3D woven structure $\varepsilon^{\prime}$ eff can be readily obtained from Equations (17) and (18):

$$
\varepsilon_{e f f}^{\prime}=\frac{\sum_{j=1}^{n} \sum_{k=1}^{\ell}\left(\sum_{i=1}^{\prime} \frac{1}{\mathrm{C}_{\mathrm{ijk}}}\right)^{-1} \cdot \sum_{i=1}^{m} d_{i}}{\varepsilon_{0} \sum_{j=1}^{n} \sum_{k=1}^{\ell} \mathrm{A}_{j k}}
$$




\section{Verification of the proposed 3D electromagnetic modelling methodology}

4.1 Comparison with the literature

The proposed methodology is compared with the prediction presented in Reference [11], where interply and intraply hybrid composites were studied. As given in Table 4, the accuracy of the dielectric constants offered by the present work is improved with errors within $5 \%$.

Table 4 Dielectric constants predicted by the present work and the literature

\begin{tabular}{cccccc}
\hline \multirow{2}{*}{ Configuration } & \multirow{2}{*}{ Experiment [11] } & \multicolumn{2}{c}{ Reference [11] } & \multicolumn{2}{c}{ Present work } \\
& & Value & Error (\%) & Value & Error (\%) \\
\hline Interply & 3.95 & 3.66 & -7.34 & 3.8091 & -3.57 \\
Intraply & 3.91 & 3.67 & -6.14 & 3.8062 & -2.66 \\
\hline
\end{tabular}

\subsection{Experiment}

\subsubsection{Sample preparation}

A 3D woven angle interlock glass fibre sample was measured for further verification. As illustrated in Figure 8, the sample consisted of four warp layers, three weft layers and the binder inserted after every three layers of the weft yarn [23]. A twofold yarn with a 1360 Tex was used in the warp and weft yarns, while a single yarn of a 680 Tex was used for the binder. The fabric counts in the warp yarns, weft yarns and binder were 3.95 ends/cm, 2.8 picks/cm and 3.0 ends/cm, respectively. The S2 glass fibres provided by AGY were infused with the epoxy resin LY564 and hardener XB 3486 from Huntsman Advanced Materials. The infusion process was done by the vacuum-assisted resin transfer moulding (VARTM) technique, then the whole assembly was moved to an oven for curing at $80{ }^{\circ} \mathrm{C}$ for eight hours. The yarn volume fractions of the warp, weft and binder were $31.21 \%, 15.83 \%$ and $3.05 \%$, respectively. The thickness of the sample was $3.03 \mathrm{~mm}$.

It is known that the dielectric constant of S2 glass fibre is 5.20 [20]. As described in Section 3, in order to predict the effective dielectric constant of the sample, the dielectric constant of the resin should be known in advance as well. Hence, a neat epoxy resin sample was fabricated for evaluation. 


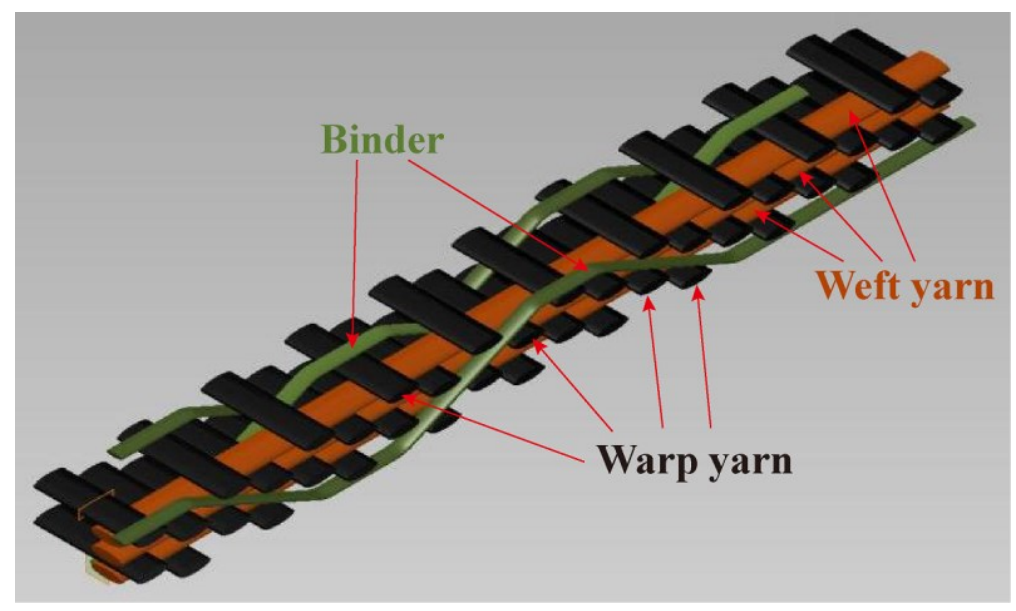

Figure 8 Schematic diagram of the 3D woven glass fibre sample

used for verification (adapted from [23])

\subsubsection{Dielectric constant measurement}

The dielectric constant measurement over X-band was performed using the transmission line technique as schematically illustrated in Figure 9 (a). An HP8510C Vector Network Analyser (VNA) was used. It was calibrated before test using the thru-reflect-line (TRL) standard [24]. A personal computer was connected to the VNA by a GPIB cable. A MATLAB ${ }^{\circledR}$ programme was developed for data acquisition and dielectric constant computation.

The test samples with the inner dimensions of the rectangular waveguide were mounted on the waveguide flange. As shown in Figure 9 (b), the binder of the 3D woven sample was along the broad dimension of the waveguide. Hence, in the modelling the warp yarns, weft yarns and binders corresponded to Case 1, Case 2 and a general case, respectively. 


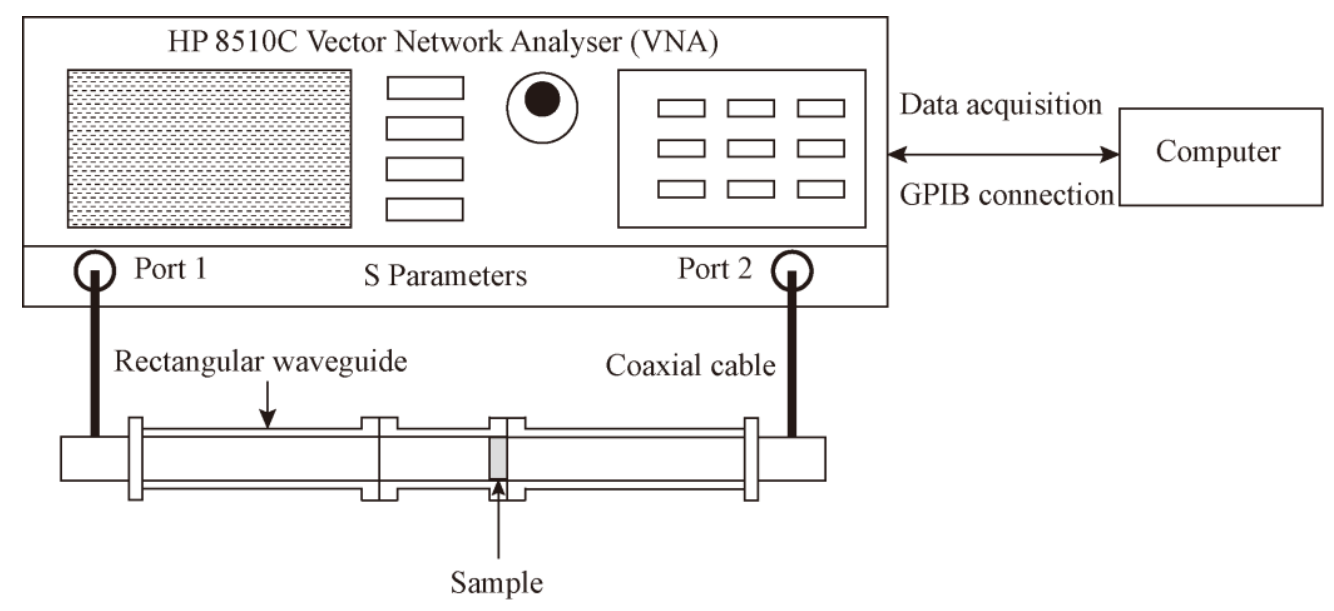

(a) Experimental setup

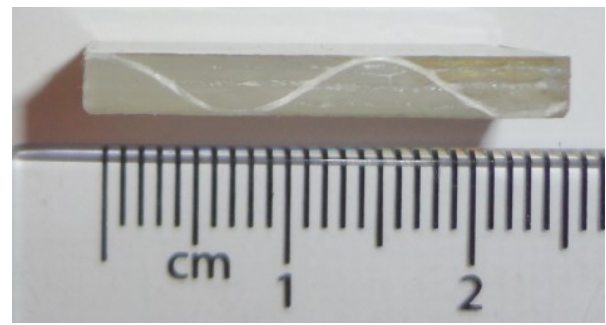

(b) Side view of the 3D woven sample under test

Figure 9 Transmission line technique for dielectric constant measurement of the 3D woven glass fibre and net resin samples

\subsubsection{Results and discussions}

$\mathrm{S}_{11}$ and $\mathrm{S}_{21}$ obtained from the test of the 3D woven glass fibre sample are presented in Figure 10.

From the measurement data, the dielectric constant is calculated using Equations (2-5). The experimental and predicted results are given in Figure 11. The average effective dielectric constant of the neat resin is approximately 2.74 , and the dielectric constant of the $3 \mathrm{D}$ woven sample remains stable at 3.75. The Wiener upper limit and Wiener lower limit are employed for comparison. The dielectric constants provided by the two limits are 3.98 and 3.60 with errors of $6.13 \%$ and $4.05 \%$, respectively. However, the dielectric constant predicted by Equation (19) is 3.85 with a smaller error of $2.67 \%$. 


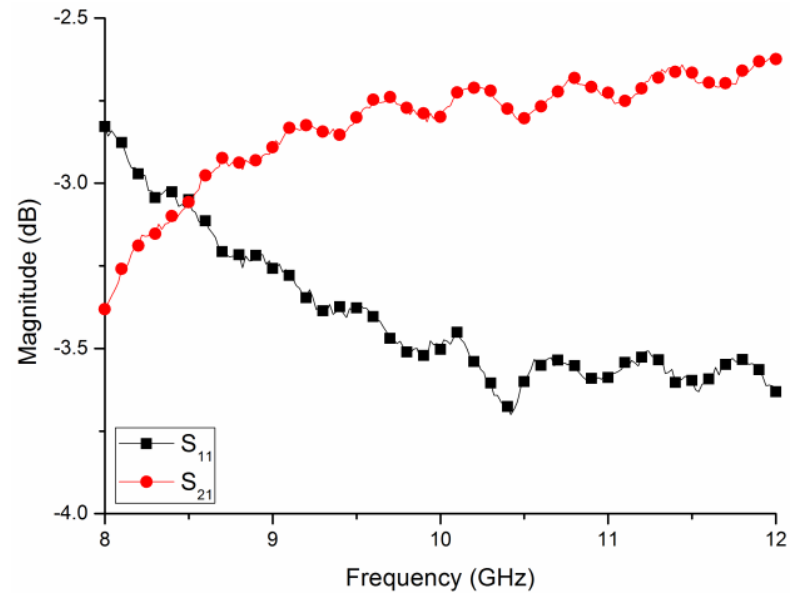

(a) Magnitudes of $S_{11}$ and $S_{21}$

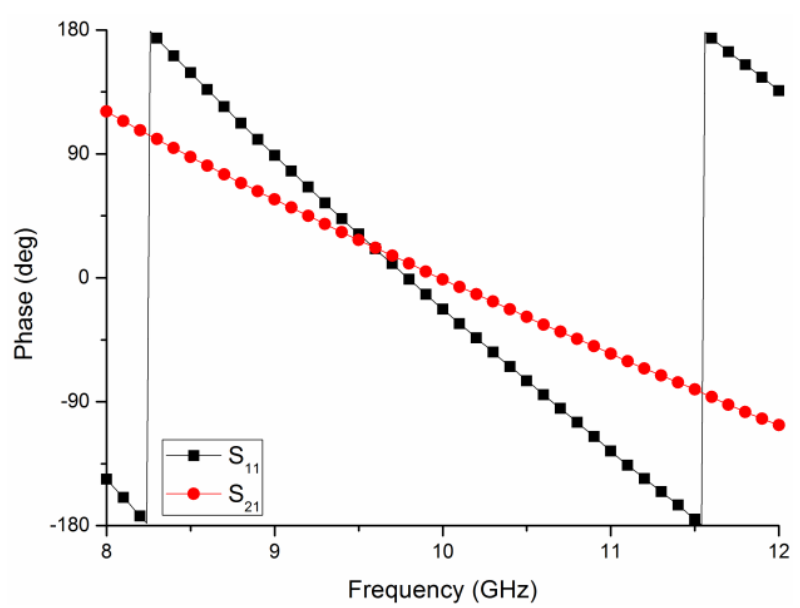

(b) Phases of $S_{11}$ and $S_{21}$

Figure $10 \mathrm{~S}_{11}$ and $\mathrm{S}_{21}$ measured from the $3 \mathrm{D}$ woven glass fibre sample over X-band

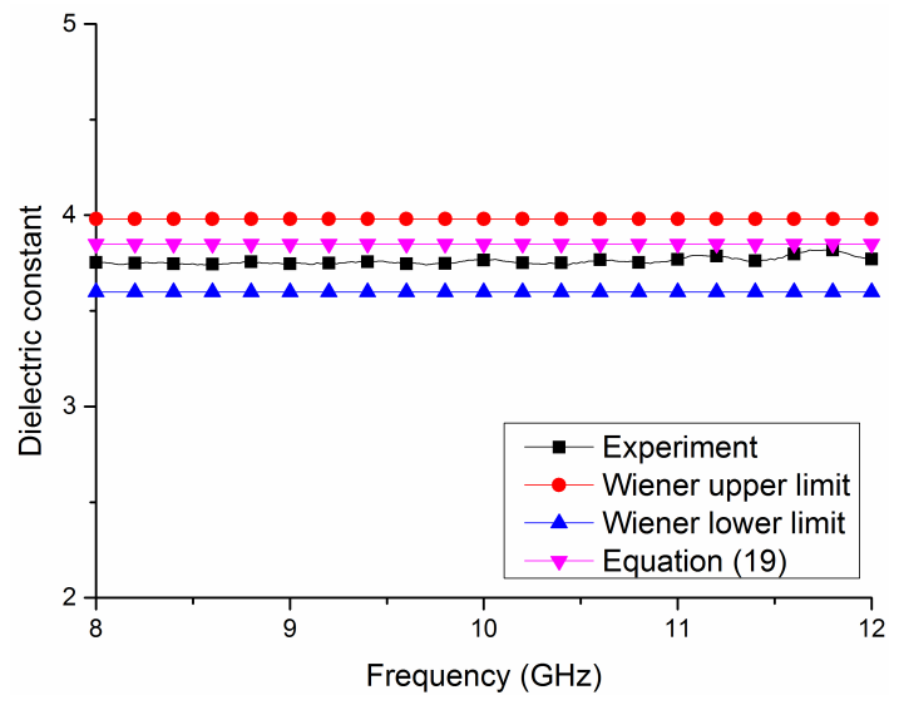

Figure 11 The measured and predicted dielectric constants of the 3D woven glass fibre sample

\section{Concluding remarks}

Permittivity characterisation of glass fibre composites is a prerequisite to modelling electromagnetic effects. In the three-dimensional weave, the fibre direction varies throughout the structure. The interaction between the electric field of incident EM waves and the fibre depends on the relative orientation and this leads to anisotropic permittivity effects. This 3D anisotropy is evaluated here for the first time using basic EM models corresponding to orthogonal fibre directions. From the numerical results the most appropriate existing mixing formula for each case is selected. 
Experimental set-ups reported in the literature and new test results presented here demonstrate improved accuracy when the anisotropy is taken into account. As a next step, this work could be extended to other types of glass fibres and other fibre architectures such as 3D braided composites.

\section{Acknowledgements}

This work was financially supported by Dean's Doctoral Scholar Award of School of Materials, The University of Manchester. The authors acknowledge the support from Paul Shaw, Mubeen Arshad and Edward Pullicino for sample preparation. The first author gratefully acknowledges Vaughan Dickinson from Huntsman Advanced Materials (Europe) for providing the epoxy resin essential for the experiments. Special thanks to Professor Prasad Potluri, Dr.Mohamed Saleh, Dr.Richard Kennon and Dr. Matthieu Gresil for their advice and many useful discussions.

\section{References}

[1] Soutis C. Fibre reinforced composites in aircraft construction. Prog Aerosp Sci 2005;41:14351. doi:10.1016/j.paerosci.2005.02.004.

[2] Li Z, Haigh A, Soutis C, Gibson A. Simulation for the impact damage detection in composites by using the near-field microwave waveguide imaging. 53rd Annu. Conf. Br. Inst. Non-Destructive Test., Manchester,UK: 2014.

[3] Li Z, Haigh A, Soutis C, Gibson A, Sloan R. Microwaves Sensor for Wind Turbine Blade Inspection. Appl Compos Mater 2017;24:495-512. doi:10.1007/s10443-016-9545-9.

[4] Potluri P, Hogg P, Arshad M, Jetavat D, Jamshidi P. Influence of fibre architecture on impact damage tolerance in 3D woven composites. Appl Compos Mater 2012;19:799-812. doi:10.1007/s10443-012-9256-9.

[5] Li Z, Haigh A, Soutis C, Gibson A, Sloan R, Karimian N. Delamination detection in composite T-joints of wind turbine blades using microwaves. Adv Compos Lett 2016;25:836. 
[6] Nemat-Nasser SC, Amirkhizi A V., Plaisted T, Isaacs J, Nemat-Nasser S. Structural composites with integrated electromagnetic functionality. In: McGowan A-MR, editor. SPIE's 9th Annu. Int. Symp. Smart Struct. Mater., International Society for Optics and Photonics; 2002, p. 237-45. doi:10.1117/12.475070.

[7] Sihvola AH. Electromagnetic Mixing Formulas and Applications. The Institution of Engineering and Technology; 1999.

[8] Bal K, Kothari V. Permittivity of woven fabrics: A comparison of dielectric formulas for airfiber mixture. IEEE Trans Dielectr Electr Insul 2010;17:881-9.

doi:10.1109/TDEI.2010.5492262.

[9] Chin WS, Lee DG. Binary mixture rule for predicting the dielectric properties of unidirectional E-glass/epoxy composite. Compos Struct 2006;74:153-62. doi:10.1016/j.compstruct.2005.04.008.

[10] Chin WS, Lee DG. Laminating rule for predicting the dielectric properties of E-glass/epoxy laminate composite. Compos Struct 2007;77:373-82. doi:10.1016/j.compstruct.2005.07.019.

[11] Yao L, Wang X, Liang F, Wu R, Hu B, Feng Y, et al. Modeling and experimental verification of dielectric constants for three-dimensional woven composites. Compos Sci Technol 2008;68:1794-9.

[12] Kharkovsky S, Zoughi R. Microwave and millimeter wave nondestructive testing and evaluation - Overview and recent advances. IEEE Instrum Meas Mag 2007;10:26-38. doi:10.1109/MIM.2007.364985.

[13] Mallick PK. Fiber-reinforced composites : materials, manufacturing, and design. 3rd ed. CRC Press; 2007.

[14] Khatavkar N, K. B. Composite materials for supersonic aircraft radomes with ameliorated radio frequency transmission-a review. RSC Adv 2016;6:6709-18. doi:10.1039/C5RA18712E. 
[15] Tischer FJ. Measurement of the wave-propagation properties of plasma in the microwave region. IRE Trans Instrum 1960;I-9:167-71. doi:10.1109/IRE-I.1960.5006909.

[16] High strength glass fibers. Technical paper. AGY.2014. Available from:http://www.agy.com/wp-content/uploads/2014/03/High_Strength_Glass_FibersTechnical.pdf

[17] Seo IS, Chin WS, Lee DG. Characterization of electromagnetic properties of polymeric composite materials with free space method. Compos Struct 2004;66:533-42. doi:10.1016/j.compstruct.2004.04.076.

[18] Chen X, Li Z, Wang H. Progressive Failure Analysis of an Open-Hole Composite Laminate by Using the S-Version Finite-Element Method. Mech Compos Mater 2014;50:279-94. doi:10.1007/s11029-014-9414-2.

[19] Marion J, Hornyak W. Physics for science and engineering. CBS college publishing; 1982.

[20] S-2 glass fiber: the high performance system solution. AGY.2004. Available from:http://www.agy.com/wp-content/uploads/2014/03/Advanced_Materials_BrochureTechnical.pdf

[21] Hu D. Development of the epoxy composite complex permittivity and its application in wind turbine blades. Queen Mary, University of London, 2010.

[22] Ansar M, Wang X, Zhou C. Modeling strategies of 3D woven composites: a review. Compos Struct 2011;93:1947-63. doi:10.1016/j.compstruct.2011.03.010.

[23] Arshad M. Damage tolerance of 3D woven composites with weft binders. University of Manchester, 2014.

[24] Haigh AD, Thompson F, Gibson AAP, Campbell GM, Fang C. Complex permittivity of liquid and granular materials using waveguide cells. Subsurf Sens Technol Appl 2001;2:42534. doi:10.1023/A:1013273102533. 